\title{
BIOBANKS AND PUBLIC HEALTH: A NEW CHALLENGE FOR PUBLIC ENGAGEMENT AND TRUST
}

\section{Luciana Caenazzo ${ }^{1,2}$, Pamela Tozzo $^{3}$}

${ }^{1}$ Department of Molecular Medicine, University of Padua, Padova, Italy

${ }^{2}$ Fondazione Lanza, Padova, Italy

${ }^{3}$ Department of Biotechnologies and Sciences of Life, Insubria University, Varese, Italy

\author{
Corresponding Author: \\ Luciana Caenazzo \\ Department of Molecular Medicine, \\ University of Padua \\ 50, Via Falloppio Str. \\ Padova, 35121 \\ Italy \\ e-mail: luciana.caenazzo@unipd.it
}

Received: September 09, 2015

Revision received: January 27, 2016

Accepted: May 30, 2016

\section{Summary}

Modern biobanks should be considered as important structural platforms for sharing samples and data, providing benefits that go beyond those of a single study. To increase the impact of biobanking on feasible strategies to protect and improve peoples' health, it could be useful to increase awareness about biobank research potential in the scientific community, as well as to promote collaboration among different fields of expertise. The basis for involvement of biobanks in public health has already been partly built up, emphasising the synergy in organising efforts and the openness to infrastructure collaboration, to ensure reliable long-term funding and resource sharing to the benefit of all. To obtain the maximum benefits possible from biobanks, which currently constitute a set of heterogeneous structures, a strategy of coordinated governance is needed, based on harmonisation of different aspects, built on the consensus of all stakeholders involved and society in general. It is important that researchers strive to understand public attitudes in this biobanking involvement so that the relationships between public trust in biobanking, donors' behaviours and support for public-health involvement in the health-care system can be better understood.

Key words: biological specimen banks, public health, trust, patient engagement

\section{Introduction}

Public health is the societal approach to protecting and promoting health and improving the well-being of communities, usually through social actions. As described in literature, it can be defined as the science and art of preventing disease, prolonging life and promoting health through organised efforts of society.

Biobanks are repositories, which assemble, store, and manage collections of human specimens and related data. While the collection of samples and data for research purposes has a long history in the educational and medical systems, their recent increase in number, size, and importance has focused the attention on the changing nature of biomedical research and relationships among investigators, research participants, and the organisations that fund and manage these entities [1]. 
With recent technological advances, the potential to open up these existing collections for new uses is starting to be realised, and new biobanks are being established. As a result, many types of biobanks have been founded for a wide range of purposes and reasons.

What distinguishes the present from the past is that the general scientific context has changed, and the scale of biobanking activities, both in terms of the quantity of samples and data, as well as the range of disease areas and institutions now involved in biobanking, have increased considerably.

Another significant change is that these collections are being configured in a way that allows the entire scientific community to use them as a resource. In fact, there is an increased need for new solutions, so that the results of research findings obtained through biobanks can be translated into health planning programs for the benefit of the general population. The results of these studies could be of paramount importance for planning effective and qualified interventions for public health priorities, designing national health strategies, and developing preventive medicine interventions.

Modern biobanks should be considered as important structural platforms for sharing samples and data, providing benefits that go beyond those of a single study. For this reason, it has been suggested that the characteristics, which should be considered when a biobank is designed and structured are adaptability, tenability, and ease of translation into other national contexts [2].

\section{Biobanking and Public Health}

In many European countries, the potential utility of biobanks in contributing to preventive and predictive medicine has been precluded by lack of widespread information on each collection, and of national and international networking, which also leads to a reduced possibility of establishing collaborative projects and research that would increase the impact of biobank research on public health [3].

To increase the impact of biobanking on feasible strategies to protect and improve people's health, it could be useful to increase awareness about biobanks' research potential in the overall scientific community, and promote collaboration among different fields of expertise.

A viable and equitable process of connecting biobank research to public health interventions should meet the highest ethical, legal, and socially appropriate standards. Similarly, biobank research should also be accompanied by structured policies to guarantee that research findings and results are useful for public health needs, and that human rights, as well as research ethical standards, are respected [4].

Public health, unlike clinical medicine, is concerned with populations rather than with individuals, and with prevention rather than with cure, so the principles of clinical medical ethics should not be simply and automatically applicable to public health, where the need to ensure the health of the general population should be balanced against avoiding abuse towards individuals. Public health research poses particular challenges in different important areas, such as anonymisation of samples and data, donors' information and consent, and the return of research results to the donors. Moreover, public health should also aim at improving the population's knowledge about scientific research, as well as at making scientific assumptions that justify the involvement of the population in research projects, in order to increase the awareness, understanding, and acceptance of biobanks' activities by the population [5-7].

\section{Ethics and Harmonisation Policies}

Establishing new biobanks implies developing new ways of conducting research, because today's research projects are frequently global, involving international teams and cross-border networks of experts from various fields from different institutions, which pool and share samples and data. This is what makes harmonization in practice essential; the aim is to place the most useful possible resources at our disposal, helping researchers in their activity, to provide a flexible framework that should not be an obstacle for the technical progress of a biobank, and offer all communities the opportunity to benefit from the results obtained. For all these reasons, biobank harmonization, carried out on a standardised ethical and legal platform, should become a key issue in the field of biobanking, both in terms of regulation and governance, with respect to peculiar challenges such as cross-border exchange and transfer of samples and valid sharing of data and results.

Biobank harmonisation initiatives have 
developed not only in technical aspects, such as specimen and data management, but also in sharing ethical and policy principles with transparency, and with the goal to apply translational science for public benefit. Harmonization undoubtedly increases the quantity of usable data and number of biospecimens to implement the development of translational research, and may help to overcome some of the historical boundaries between clinical and basic research.

At the beginning of the development of biobanking ethical and legal attention was centered on donors' involvement, and particularly on the topics of informed consent, privacy protection, proprietorship of samples, return of results, and risk of stigmatisation. Considering these topics still important, nowadays we believe that, for biobanks to be useful in public health, improvement in ethical dialogue is necessary to accommodate broader social and political perspectives in healthcare policies. In fact even, if until recently the debate on shared ethical principles that foster international scientific collaborations has been paramount, the principle of "public goods", achieved by altruism, solidarity and data sharing needs to be promoted, moving the ethical discussion on biobanks from an individualistic to a societal perspective, while maintaining the necessary attention to respect of traditional ethical principles $[4,8]$.

\section{Public Engagement and Trust in Biobanks}

Developing people's trust in biobanks research is essential to maintain and increase their activities. This will facilitate sample donation to biobanks for the benefit of all individuals. In this sense, it is essential to promote educational strategies involving the media and society in general to encourage sample donation, as well as public engagement in biobanking. Furthermore, it should be the duty of researchers involved in biobanks to act responsively to maintain transparency and informative efficacy on their activities, to increase public trust and engagement in donation and biobanking research.

Public trust may concern the expectations that one individual is an entity and it involves acceptance of risk and exposure to vulnerability. It means that individuals have expectations about the actions of the entity involved and they are supposed to take their individual risk undertaking these actions. Viewed from this perspective, trust in biobanking governance may serve to increase one's willingness to support biobanks activity by encouraging sample donation and supporting public encouragement in the development of biobanking technologies and research.

As stated in literature, "for the adequate development of basic rights and shared values something else is essential: participatory selfrule. Every individual should be able to engage in public debate, and actively take part in social and community life to foster and promote the important values and liberties" $[9,10]$.

Regarding biobanking, sample donation does not seem difficult, if we consider that donors see human samples being kept rather than discarded, and see them becoming useful for research. Since donation might benefit others and contribute to a generalised system of solidarity, the importance of the human body is highlighted and the attitude towards donation is improved.

This process might also facilitate giving informed consent more trustfully and willingly, granting, in this context, that donation permission is nothing more than an automatic response [8].

Concerning this topic, for example, the results reported by Morrell [11] show that donors had a high level of willingness to donate residual tumour tissue for research. Donation was often considered "not a big deal" given that it involved the use of tissues, which would be discarded anyway and was perceived as involving little or no credible risk. Despite the sense that donation was "a lot of fuss about nothing," donors expressed considerable satisfaction at the thought that their donation might benefit others and contribute to a "generalised system of reciprocity".

Including biobanks in the health-care system could be an important step towards their financing, developing sustainability through stable funding, and their emergence as a systematic infrastructure of primary importance in public health. On the other hand, it is important that biobanks are established and managed so that they take into account the needs, development plans, and future programs of public health, understanding, therefore, the research needs, which are more functional to the protection of global health. Both the benefits of scientific knowledge implementation and the benefits of individuals linked to the knowledge of their health status, show that biobanks may have a fundamental role within the public system of 
health-care.

It is essential that operators, sponsors, and researchers of biobanks cooperate with health policy-makers in order to ensure integrated and structured governance, including regulatory aspects, technical aspects, and quality assurance aspects, to meet the current and future needs at both individual and societal level.

\section{Conclusions}

Biobanking may represent a valuable tool for innovation in public health and production of scientific knowledge, acquiring adequate resources and the ability to integrate the different biobanks in national and international networks. The perception of general needs of individuals and society to be informed and made positive is crucial for enhancing the productivity and scientific usefulness of biobanks in public health.

In this perspective, the promotion of educational strategies involving the media and society in general to encourage sample donation, as well as biobanking engagement, is critical not only to ensure an appropriate use of available resources, but also in achieving a more structured maintainance of biobanks.

The basis for involvement of biobanks in public health has already been partly built up, emphasising the synergy in organising efforts and the openness to infrastructure collaboration, to ensure reliable long-term funding and resource sharing for the good of all. To obtain the maximum benefits possible from biobanks, which currently constitute a set of heterogeneous structures, a strategy of coordinated governance is needed, based on harmonisation of structural, technological, scientific, social, legal, and ethical aspects, built on the consensus of all stakeholders involved, and society at large.

One of the possible strategies for building the scientific, social, ethical, and political framework to develop biobanking for public health should be elucidating and building trust on biobanking activities and purposes by the media, the public, policymakers, and health-care strategists, and by supporting the recognition of the scientific contributions given by researchers involved in biobanks.

There is an increased need for new solutions in order to translate into health planning programs the results of research findings for the benefit of the general population. The results of these studies could be of paramount importance for planning effective and qualified interventions for public health priorities, for designing national health strategies, and for developing preventive medicine interventions.

It is important that researchers strive to understand public attitudes in this biobanking involvement so that the relationships between public trust in biobanking, donor behaviours and support for public-health involvement in the health-care system can be better understood.

\section{References}

1. Watson PH, Wilson-McManus JE, Barnes RO, Giesz SC, Png A, Hegele RG, et al. Evolutionary concepts in biobanking - the BC BioLibrary. J Transl Med. 2009; 7:95.

2. Harris JR, Burton P, Knoppers BM, Lindpaintner $\mathrm{K}$, Bledsoe $\mathrm{M}$, Brookes AJ, et al. Toward a roadmap in global biobanking for health. Eur $\mathbf{J}$ Hum Genet. 2012;20(11):1105-11.

3. Bravo E, Napolitano M, Santoro F, Belardelli F, Federic A. The Italian Hub of Population Biobanks as a potential tool for improving public health stewardship. Biopreserv Biobank. 2013;11(3):173-5.

4. Budimir D, Polasek O, Marusić A, Kolcić I, Zemunik T, Boraska V, et al. Ethical aspects of human biobanks: a systematic review. Croat Med J. 2011;52(3):262-79.

5. Kass NE. An ethics framework for public health. Am J Public Health. 2001;91(11):1776-82.

6. O'Doherty KC, Hawkins AK, Burgess MM. Involving citizens in the ethics of biobank research: informing institutional policy through structured public deliberation. Soc Sci Med. 2012;75(9):1604-11.

7. Nicol D, Critchley C. Benefit sharing and biobanking in Australia. Public Underst Sci. 2012;21(5):534-55.

8. Caenazzo L, Tozzo P, Pegoraro R. Biobanking research on oncological residual material: a framework between the rights of the individual and the interest of society. BMC Med Ethics. 2013;14:17.

9. Hoedemaekers R, Gordijn B, Pijnenburg M. Does an appeal to the common good justify individual sacrifices for genomic research? Theor Med Bioeth. 2006;27(5):415-31.

10. Hoedemaekers R, Gordijn B, Pijnenburg M. Solidarity and justice as guiding principles in genomic research. Bioethics. 2007;21(6):342-50.

11. Morrell B, Lipworth W, Axler R, Kerridge I, Little M. Cancer as rubbish: donation of tumor tissue for research. Qual Health Res. 2011;21(1):75-84. 